\title{
OSIRIS WAVELETS AND SET WAVELETS
}

\author{
GUY BATTLE
}

Received 21 April 2004

An alternative to Osiris wavelet systems is introduced in two dimensions. The basic building blocks are continuous piecewise linear functions supported on equilateral triangles instead of on squares. We refer to wavelets generated in this way as Set wavelets. We introduce a Set wavelet system whose homogeneous mode density is $2 / 5$. The system is not orthonormal, but we derive a positive lower bound on the overlap matrix.

\section{Introduction}

The decomposition of a scalar field into continuous piecewise-linear wavelets is the central approach in a new hierarchical modeling program $[1,2,3,4,5]$ for studying the critical behavior of certain classical systems in equilibrium statistical mechanics (such as the classical dipole gas and the Ginzburg-Landau spin system). Naturally, such a wavelet decomposition must be based on a multiscale triangulation of space, and in two dimensions we have been generating it $[4,5]$ from the triangulation of the next-to-minimum-scale square given by the diagram in Figure 1.1. The notion of orthogonality between continuous piecewise-linear functions that is natural to the modeling is based on the Sobolev inner product

$$
\langle f, g\rangle=\int \nabla f \cdot \nabla g
$$

where the functions are real-valued. Choosing the unit scale as the minimum scale, consider the two functions given in $[0,2]^{2}$ by the gradient diagrams in Figures $1.2(\mathrm{a})$ and 1.2(b) where each arrow represents a constant gradient in its triangle. The set of all $2 \mathbb{Z}^{2}$ translations and $2^{r}$-scalings thereof for all $r \in \mathbb{Z}$ is Sobolev-orthogonal, as was easily verified in [4]. One can certainly normalize these functions with respect to the Sobolev norm given by

$$
\|f\|^{2}=\int|\nabla f|^{2}
$$

Copyright (C) 2004 Hindawi Publishing Corporation Journal of Applied Mathematics 2004:6 (2004) 495-528 2000 Mathematics Subject Classification: 42C40, 47N99, 82B28 URL: http://dx.doi.org/10.1155/S1110757X04404070 


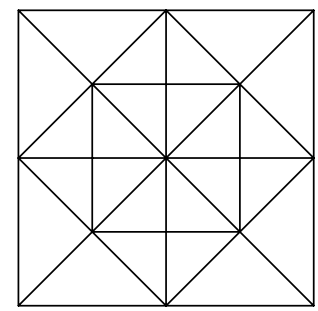

Figure 1.1

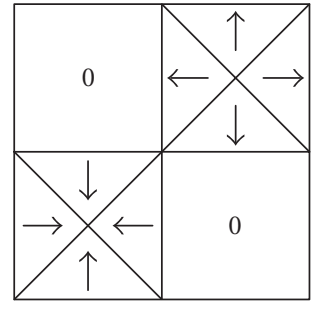

(a)

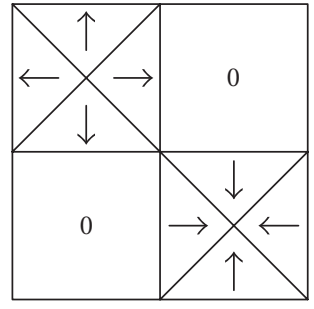

(b)

Figure 1.2

but the orthonormal set is not a basis. We measure this lack of completeness with the homogeneous mode density $[1,4]$.

Indeed, consider the set of all such wavelets of scale greater than or equal to 1 supported in the region $\left[0,2^{N}\right]^{2}$. The largest-scale wavelets are of scale $2^{N-1}$, and there is a pair of them. Clearly, there are four pairs of $2^{N-2}$-scale wavelets, sixteen pairs of $2^{N-3}$ scale wavelets, ..., and $4^{N-1}$ pairs of unit-scale wavelets. The total number of wavelets is therefore

$$
2 \sum_{k=0}^{N-1} 4^{k}=\frac{2}{3}\left(4^{N}-1\right) .
$$

How does this compare to the number of degrees of freedom generated by this multiscale scheme? The triangulation in this case is simply the triangulation of each unit square given by the diagram in Figure 1.3. The dimension of this space of continuous piecewise-linear functions is precisely the number of vertices in the triangulation, and these vertices are either centers of unit squares or their corners. Obviously, there are $4^{N}$ such centers and $\left(2^{N}+1\right)^{2}$ vertices that are corners of these squares. The homogeneous mode density of the system of wavelets is defined as the infinite-volume limit of the ratio of the number of wavelets to the number of degrees of freedom-in this case, the $N=\infty$ limit of the ratio of the number of wavelets to the number of vertices. Thus 


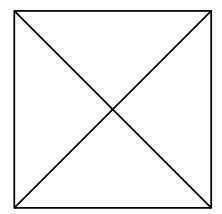

Figure 1.3

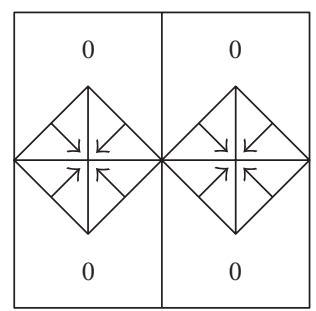

(a)

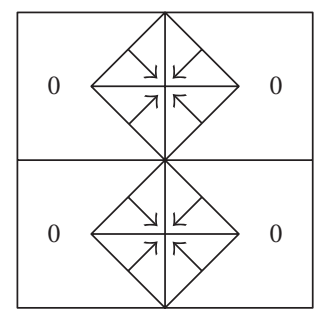

(b)

Figure 1.4

we calculate

$$
\lim _{N \rightarrow \infty} \frac{(2 / 3)\left(4^{N}-1\right)}{4^{N}+\left(2^{N}+1\right)^{2}}=\frac{1}{3} .
$$

In $[1,2,3,4,5]$ we have referred to these wavelets as Osiris wavelets, and the lack of completeness may be regarded as a hierarchical cutoff on the scalar field. Notice that every wavelet in our system is supported by its localization block, and this is the property that the term "hierarchical" refers to in the context of statistical mechanics. The idea introduced in [2] is to replace the hierarchical cutoff with a hierarchical approximation by scaling the Gaussian part of the functional integration with scale factor equal to the square root of the homogeneous mode density. For this particular orthonormal set, this is equivalent to a 3-fold replication of wavelet amplitudes in the functional integration [2].

One can enhance this system of wavelets-preserving the hierarchical property-by sacrificing some of the multiscale Sobolev orthogonality. We introduce two additional functions in $[0,2]^{2}$ given by the gradient diagrams in Figures 1.4(a) and 1.4(b). These functions are obviously Sobolev-orthogonal to the other two, but not to all of their copies on the next scale down. On the other hand, we proved in [4] that the Sobolev overlap matrix has a positive lower bound. We also showed that the homogeneous mode density of this enlarged system of Osiris wavelets is just 4/9. 


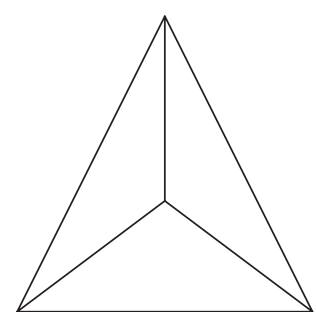

(a) +

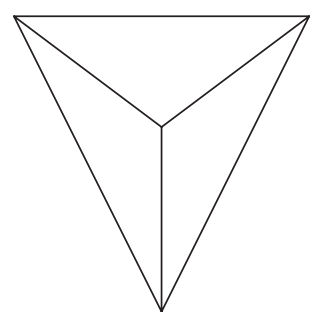

(b) -

Figure 1.5

In this paper, we investigate the option of basing a system of continuous piecewiselinear wavelets on equilateral triangles instead of on squares. We can obviously tile the plane with unit equilateral triangles (i.e., whose sides are one unit in length) having either a south side and a north vertex or a north side and a south vertex. We will refer to the former as a + triangle and to the latter as a - triangle. Let $\Delta^{+}$denote the + triangle in the first quadrant with one vertex at the origin - that is, with vertices $(0,0),(1,0)$, and $(1 / 2,(1 / 2) \sqrt{3})$. Let $\Delta^{-}$denote the triangle with vertices $(1,0),(1 / 2,(1 / 2) \sqrt{3})$, and $(3 / 2,(1 / 2) \sqrt{3})$. Clearly, all of the \pm triangles are the translates

$$
\Delta^{ \pm}+v_{0} \hat{i}+v_{1}\left(\frac{1}{2} \hat{i}+\frac{1}{2} \sqrt{3} \hat{j}\right), \quad v \in \mathbb{Z}^{2}
$$

To each such triangle we associate a triangulation given by the diagrams in Figures 1.5(a) and $1.5(\mathrm{~b})$. Each $120^{\circ}$ isosceles triangle in this division of an equilateral triangle is a primary basic triangle. To avoid serious multiscale orthogonality problems in our wavelet construction, we must have the centers of these triangles coincide with vertices of their copies on the next scale down. Since the center of a unit equilateral triangle is a distance $1 / 3$ from each side, the scale factor must therefore be 3 instead of 2 .

Now consider two successive scales, where the larger-scale equilateral triangle is, say, $\mathrm{a}+$ triangle. The triangulation is given by the diagram in Figure 1.6 where the dashed lines determine the larger-scale division. Notice that twelve right triangles in particular have been formed by the two scales of division. Such triangles are secondary basic triangles, and they appear in groups of four, with each group forming a rhombus. Now a primary basic function in this master triangle is supported by any one of the nine equilateral sub-triangles and is given by the gradient diagrams in Figures 1.7(a) and 1.7(b). as the case may be. Each of the three rhombuses in the master triangle supports a secondary basic function, and these functions are given by the gradient diagrams in Figures 1.8(a), 1.8(b), and 1.8(c). We will label these functions 1st, 2nd, and 3rd, respectively, with 


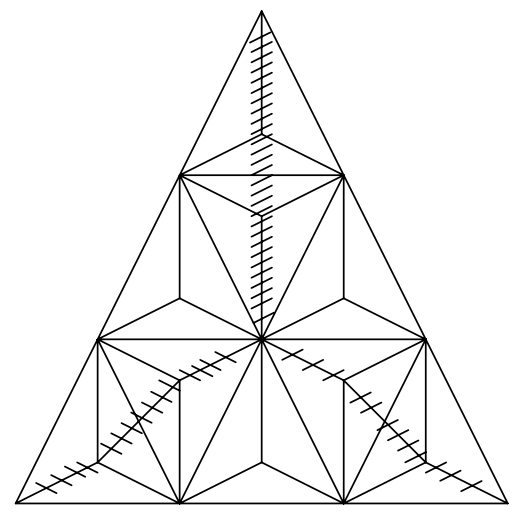

Figure 1.6

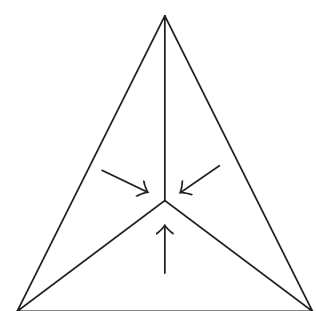

(a) +

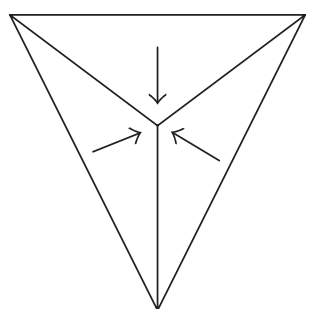

(b) -

Figure 1.7

the understanding that the master triangle is a + triangle. For the purpose of labeling the primary basic functions in the master triangle, we choose the schemes in Figures 1.9(a) and 1.9(b) for a + triangle and a - triangle, respectively. In the latter case, the secondary basic functions are given by the same gradient diagrams as in Figure 1.8. The only difference is that the 1st is now northwest, the 2 nd is now south, and the 3rd is now northeast. We regard both types of functions as unit-scale basic functions if each side of the master triangle is 3 units long. Let $\varphi_{i}^{ \pm}$denote the $i$ th primary unit-scale basic function and $\eta_{i}^{ \pm}$ the $i$ th secondary unit-scale basic function in the respective master triangles $3 \Delta^{ \pm}$, where the gradient vectors are unit vectors.

We are now ready to define Set wavelets. The unit-scale Set wavelets are combinations of unit-scale basic functions in the same 3-scale master triangle, and that triangle is the support triangle of those particular unit-scale wavelets. In the respective support triangles 


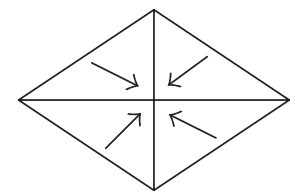

(a) (North).

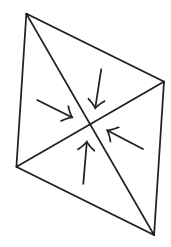

(b)

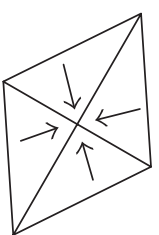

(c) (Southeast).

(Southwest).

Figure 1.8

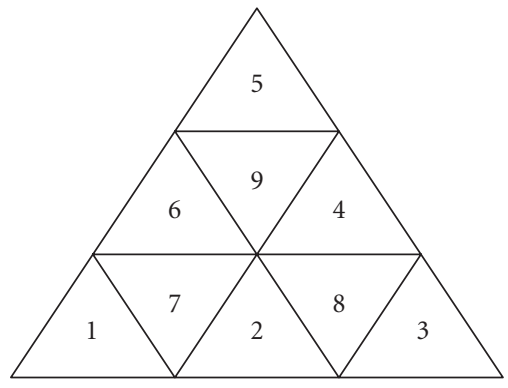

(a)

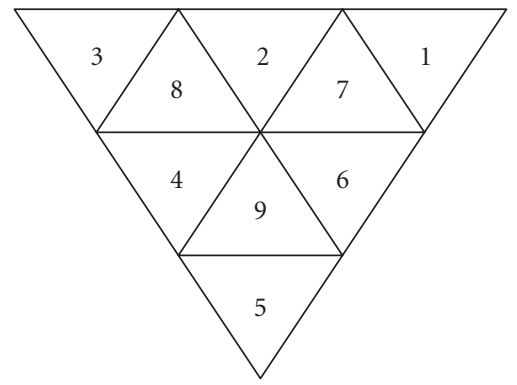

(b)

Figure 1.9

$3 \Delta^{ \pm}$we define the unit-scale Set wavelets as follows:

$$
\begin{aligned}
& \Psi^{1 \pm}=3^{-1 / 4} \sqrt{2}\left(\varphi_{1}^{ \pm}-\varphi_{7}^{ \pm}\right), \\
& \Psi^{2 \pm}=3^{-1 / 4} \sqrt{2}\left(\varphi_{3}^{ \pm}-\varphi_{8}^{ \pm}\right), \\
& \Psi^{3 \pm}=3^{-1 / 4} \sqrt{2}\left(\varphi_{5}^{ \pm}-\varphi_{9}^{ \pm}\right), \\
& \Psi^{4 \pm}=\beta \eta_{1}^{ \pm}-\alpha \varphi_{2}^{ \pm}, \\
& \Psi^{5 \pm}=\beta \eta_{2}^{ \pm}-\alpha \varphi_{4}^{ \pm}, \\
& \Psi^{6 \pm}=\beta \eta_{3}^{ \pm}-\alpha \varphi_{6}^{ \pm},
\end{aligned}
$$

where we have normalized these wavelets with respect to the Sobolev norm. In particular,

$$
\frac{1}{4} \sqrt{3} \alpha^{2}+\frac{1}{2 \sqrt{3}} \beta^{2}=1
$$


but we have yet to fix $\alpha$ and $\beta$. All of the unit-scale Set wavelets have the form

$$
\Psi_{\nu}^{\prime \pm}(\vec{x})=\Psi^{\prime \pm}\left(\vec{x}-3 v_{0} \hat{i}-3 v_{1}\left(\frac{1}{2} \hat{i}+\frac{1}{2} \sqrt{3} \hat{j}\right)\right), \quad v \in \mathbb{Z}^{2}
$$

while all of the $3^{r}$-scale Set wavelets for arbitrary $r \in \mathbb{Z}$ have the form

$$
\Psi_{r \nu}^{\prime \pm}(\vec{x})=\Psi_{\nu}^{\prime \pm}\left(3^{-r} \vec{x}\right), \quad \nu \in \mathbb{Z}^{2}
$$

The twelve wavelets $\Psi^{\prime \pm}$ are the mother wavelets which generate this system through these scalings and skewed translations in the plane. For $\iota=1,2,3$ (resp., $\iota=4,5,6$ ) we refer to the wavelets $\Psi_{r v}^{\prime \pm}$ as Set wavelets of the first kind (resp., second kind).

As we will see in Section 2, this system of wavelets is not orthonormal, but we will fix $\beta=\alpha \sqrt{3}$ to endow it with a certain orthogonality property. Consider the overlap matrix, which is given by

$$
S_{r r^{\prime} ; \nu \nu^{\prime} ; l l^{\prime} ; \pm \pm^{\prime}}=\int \nabla \Psi_{r \nu}^{l \pm} \cdot \nabla \Psi_{r^{\prime} \nu^{\prime}}^{\iota^{\prime} \pm^{\prime}}
$$

The aim of this paper is to obtain a positive lower bound on $S$. We calculate the elements of this matrix in Section 2, but we can verify here and now that

$$
S_{r r ; v v^{\prime} ; l^{\prime} ; \pm \pm^{\prime}}=\delta_{\gamma v^{\prime}} \delta_{l l^{\prime}} \delta_{ \pm \pm^{\prime}}
$$

that is, that Set wavelets on the same scale are orthonormal. We choose the unit scale without loss and note that

$$
\begin{aligned}
& \operatorname{int} \operatorname{supp} \Psi_{\nu}^{l^{ \pm}} \cap \operatorname{int} \operatorname{supp} \Psi_{\nu^{\prime}}^{l^{\prime} \pm^{\prime}}=\varnothing, \quad v^{\prime} \neq \nu, \\
& \operatorname{intsupp} \Psi_{\nu}^{l+} \cap \operatorname{int} \operatorname{supp} \Psi_{\nu}^{l^{\prime}-}=\varnothing,
\end{aligned}
$$

so the verification of $(1.11)$ is reduced to showing that

$$
\int \nabla \Psi_{\nu}^{\prime \pm} \cdot \nabla \Psi_{\nu}^{\iota^{\prime} \pm}=0, \quad \iota \neq \iota^{\prime}
$$

Again without loss, we set $v=0$ and choose $3 \Delta^{+}$over $3 \Delta^{-}$. Now clearly,

$$
\begin{aligned}
& \left\{\operatorname{int} \operatorname{supp} \Psi^{\iota+}: \iota=1,2,3\right\} \\
& \left\{\operatorname{int} \operatorname{supp} \Psi^{\iota+}: \iota=4,5,6\right\}
\end{aligned}
$$

are both collections of disjoint sets. Moreover,

$$
\begin{array}{ll}
\operatorname{int} \operatorname{supp} \Psi^{1+} \cap \operatorname{int} \operatorname{supp} \Psi^{2+}=\varnothing, & \iota \neq 5, \\
\operatorname{int} \operatorname{supp} \Psi^{2+} \cap \operatorname{int} \operatorname{supp} \Psi^{\iota+}=\varnothing, & \iota \neq 6, \\
\operatorname{int} \operatorname{supp} \Psi^{3+} \cap \operatorname{int} \operatorname{supp} \Psi^{\iota+}=\varnothing, & \iota \neq 4,
\end{array}
$$




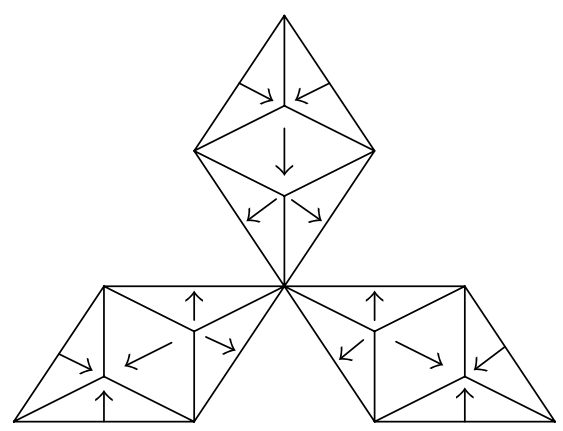

Figure 1.10

so we only need to examine the inner product of $\Psi^{1+}$ (resp., $\Psi^{2+}, \Psi^{3+}$ ) with $\Psi^{5+}$ (resp., $\left.\Psi^{6+}, \Psi^{4+}\right)$. Obviously,

$$
\begin{aligned}
& \operatorname{int} \operatorname{supp} \Psi^{1+} \cap \operatorname{int} \operatorname{supp} \Psi^{5+}=\operatorname{int} \operatorname{supp} \eta_{2}^{+}, \\
& \operatorname{int} \operatorname{supp} \Psi^{2+} \cap \operatorname{int} \operatorname{supp} \Psi^{6+}=\operatorname{int} \operatorname{supp} \eta_{3}^{+}, \\
& \operatorname{int} \operatorname{supp} \Psi^{3+} \cap \operatorname{int} \operatorname{supp} \Psi^{4+}=\operatorname{int} \operatorname{supp} \eta_{1}^{+}
\end{aligned}
$$

Moreover, the gradient diagram for $\Psi^{1+}, \Psi^{2+}$, and $\Psi^{3+}$ together in their support triangle is just as in Figure 1.10. Note that $\nabla \Psi^{1+}$ (resp., $\nabla \Psi^{2+}, \nabla \Psi^{3+}$ ) is constant on supp $\eta_{2}^{+}$(resp., $\left.\operatorname{supp} \eta_{3}^{+}, \operatorname{supp} \eta_{1}^{+}\right)$. On the other hand,

$$
\int \nabla \eta_{l}^{+}=0
$$

so it follows that

$$
\begin{aligned}
& \int \nabla \Psi^{1+} \cdot \nabla \Psi^{5+}=\beta \int \nabla \Psi^{1+} \cdot \nabla \eta_{2}^{+}=0, \\
& \int \nabla \Psi^{2+} \cdot \nabla \Psi^{6+}=\beta \int \nabla \Psi^{2+} \cdot \nabla \eta_{3}^{+}=0 \\
& \int \nabla \Psi^{3+} \cdot \nabla \Psi^{4+}=\beta \int \nabla \Psi^{3+} \cdot \nabla \eta_{1}^{+}=0 .
\end{aligned}
$$

This concludes the proof of (1.11).

This system of wavelets is not complete. To calculate the homogeneous mode density, we consider the equilateral triangle $3^{N} \Delta^{+}$. It is the support triangle for six $3^{N-1}$-scale wavelets; it also contains nine $3^{N-1}$-scale equilateral triangles, each one of which is the support triangle for six $3^{N-2}$-scale wavelets. Further, it contains 81 support triangles for $3^{N-3}$-scale wavelets, 729 support triangles for $3^{N-4}$-scale wavelets, $\ldots, 9^{N-1}$ support triangles for unit-scale wavelets. The total number of wavelets in $3^{N} \Delta^{+}$is exactly

$$
6 \sum_{k=0}^{N-1} 9^{k}=\frac{3}{4}\left(9^{N}-1\right)
$$


How many vertices are involved in the total triangulation? Consider a $3^{k}$-scale equilateral triangle with $k \geq 1$. Each vertex of this triangle is a vertex of some unit-scale equilateral triangle, and so is the center. Thus, all vertices of these two types are accounted for by the centers and vertices of the $9^{N}$ unit-scale equilateral triangles in $3^{N} \Delta^{+}$. Obviously, the number of the former is just $9^{N}$, while the number of the latter is just

$$
\sum_{n=1}^{3^{N}+1} n=\frac{\left(3^{N}+1\right)\left(3^{N}+2\right)}{2}
$$

The remaining type of vertex is the center of some $3^{k-1}$-scale rhombus in some $3^{k}$-scale equilateral triangle for some $k \geq 1$. As we have already discussed, there are three such rhombuses to such an equilateral triangle. Thus $3^{N} \Delta^{+}$contains three $3^{N-1}$-scale rhombuses, but it also contains

(1) nine equilateral triangles, each containing three $3^{N-2}$-scale rhombuses,

(2) eighty-one equilateral triangles, each containing three $3^{N-3}$-scale rhombuses,

$(N-1) 9^{N-1}$ equilateral triangles, each containing three unit-scale rhombuses.

Therefore, the total number of rhombus centers is precisely

$$
3 \sum_{k=0}^{N-1} 9^{k}=\frac{3}{8}\left(9^{N}-1\right) .
$$

In summary, the total number of vertices is given by

$$
9^{N}+\frac{\left(3^{N}+1\right)\left(3^{N}+2\right)}{2}+\frac{3}{8}\left(9^{N}-1\right)=\frac{15}{8} 9^{N}+\frac{1}{2} 3^{N+1}+\frac{5}{8},
$$

and so the homogeneous mode density is the limit

$$
\lim _{N \rightarrow \infty} \frac{(3 / 4)\left(9^{N}-1\right)}{(15 / 8) 9^{N}+(1 / 2) 3^{N+1}+5 / 8}=\frac{2}{5} .
$$

In the next section, we investigate Sobolev orthogonality properties of the system of Set wavelets and calculate the nonzero elements of the overlap matrix $S$. The key calculation will involve Sobolev inner products between 3-scale wavelets supported by $9 \Delta^{+}$and unit-scale wavelets whose support triangles lie in $9 \Delta^{+}$. With the nine 3 -scale equilateral triangles labeled as in Figure 1.9(a), we will adopt the special notation $\Psi_{0 \mu}^{\iota}$ for the $\iota$ th 
unit-scale wavelet whose support triangle is the $\mu$ th triangle. Thus

$$
\begin{aligned}
& \Psi_{01}^{\iota}=\Psi^{l+}, \\
& \Psi_{02}^{l}=\Psi_{(1,0)}^{l+} \text {, } \\
& \Psi_{03}^{l}=\Psi_{(2,0)}^{l+} \text {, } \\
& \Psi_{04}^{l}=\Psi_{(1,1)}^{l+} \text {, } \\
& \Psi_{05}^{\iota}=\Psi_{(0,2)}^{\iota+} \text {, } \\
& \Psi_{06}^{l}=\Psi_{(0,1)}^{l+} \text {, } \\
& \Psi_{07}^{\iota}=\Psi^{l-} \text {, } \\
& \Psi_{08}^{\iota}=\Psi_{(1,0)}^{\iota-}, \\
& \Psi_{09}^{\iota}=\Psi_{(0,1)}^{l-} \text {. }
\end{aligned}
$$

In Section 3, we reduce the goal of the paper to the study of nine $6 \times 6$ matrices. In Section 4, we use these matrices to show that

$$
S \geq 1-\frac{1}{12} \sqrt{26}-\frac{1}{36} \sqrt{42} .
$$

This result also requires a standard operator lemma $[3,6]$, which we state here.

Lemma 1.1. Let $X$ be a bounded operator on a Hilbert space such that

$$
\left\|X^{N}\right\| \leq a^{N-1} b
$$

for all $N$ and fixed $a, b>0$. Then

$$
\left\|X+X^{*}\right\| \leq a+b
$$

\section{Sobolev orthogonality properties}

Although the system of Set wavelets is not orthonormal, it has certain orthogonality properties that are needed to establish a positive lower bound on the overlap matrix. Our first observation is a very obvious one.

Orthogonality Property 2.1. If the support of a basic function is contained in a basic triangle of a larger-scale basic function, then the functions are Sobolev-orthogonal.

Consider an $r$-level Set wavelet of the first kind, and assume that it is a southwest wavelet without loss of generality-which also means that we have chosen a + triangle for its support triangle. The wavelet is given by the diagram in Figure 2.1 and we wish to compare it to an arbitrary s-level Set wavelet for $s \geq r+2$. By Orthogonality Property 2.1, the wavelets are orthogonal unless a line associated with the larger-scale wavelet divides the support triangle of the given wavelet. In that case, there is only one such line, and it belongs to one of the two basic functions comprising the larger-scale wavelet. This is a consequence of the assumption that the difference in scales is at least two levels. The only possibilities are illustrated by the diagrams in Figures 2.2(a), 2.2(b), and 2.2(c). In 


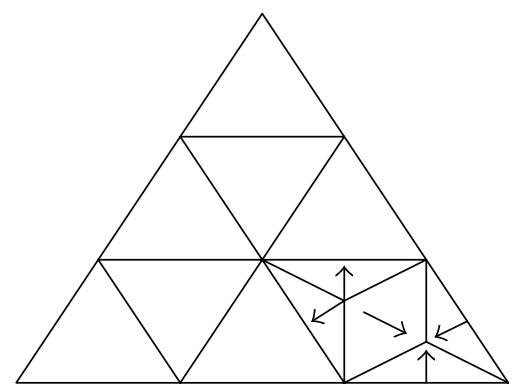

Figure 2.1

the first two cases, the line does not divide the support of the given wavelet, so Orthogonality Property 2.1 still applies. Only the third case falls outside the domain of that property, and it yields an inner product with the decomposition given by the diagrams in Figures 2.3(a) and 2.3(b). Consider the contribution given by the first diagram and denote the constant gradient of the s-level basic function on that side of the line by $\vec{v}$. By our discussion in the introduction, the left, middle, and right arrows in the diagram represent the gradients

$$
\begin{gathered}
\vec{v}_{L}=3^{-r-1 / 4} \sqrt{2}\left(-\frac{1}{2} \sqrt{3} \hat{i}-\frac{1}{2} \hat{j}\right) \\
\vec{v}_{M}=3^{-r-1 / 4} \sqrt{2}\left(\frac{1}{2} \sqrt{3} \hat{i}-\frac{1}{2} \hat{j}\right) \\
\vec{v}_{R}=3^{-r-1 / 4} \sqrt{2} \hat{j}
\end{gathered}
$$

respectively. Moreover, each triangle has area $(1 / 4) 3^{2 r-1 / 2}$, so this contribution to the Sobolev inner product is just

$$
\frac{1}{4} 3^{2 r-1 / 2} \vec{v}_{L} \cdot \vec{v}+\frac{1}{4} 3^{2 r-1 / 2} \vec{v}_{M} \cdot \vec{v}+\frac{1}{4} 3^{2 r-1 / 2} \vec{v}_{R} \cdot \vec{v}=\frac{1}{4} 3^{r-1 / 2} \vec{v} \cdot\left(\vec{v}_{L}+\vec{v}_{M}+\vec{v}_{R}\right) .
$$

Since

$$
\vec{v}_{L}+\vec{v}_{M}+\vec{v}_{R}=0
$$

this contribution vanishes. The same reasoning shows that the other contribution also vanishes, so we have established orthogonality in this case as well.

Now suppose the $r$-level wavelet is a Set wavelet of the second kind. Is it orthogonal to an arbitrary $s$-level Set wavelet for $s \geq r+2$ ? Without loss, we assume that it is a southwest wavelet, so for this kind of piecewise linear function we have the gradient diagram in Figure 2.4. The cases for the larger-scale wavelet are still covered by the diagrams of Figure 2.2, and Orthogonality Property 2.1 still applies to the first two cases. In the third case, the inner product decomposes into the contributions given by the diagrams in Figures 2.5(a) and 2.5(b). Again, consider the contribution given by the first diagram and denote the constant gradient of the s-level basic function on that side of the line by $\vec{v}$. 


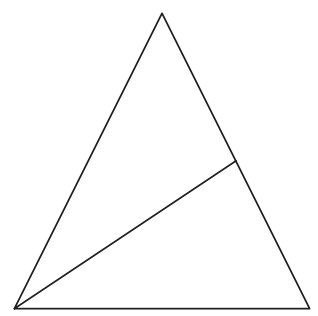

(a)

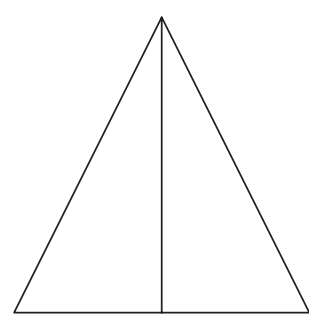

(b)

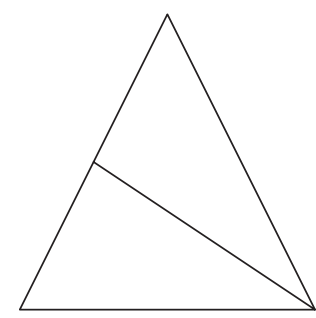

(c)

Figure 2.2

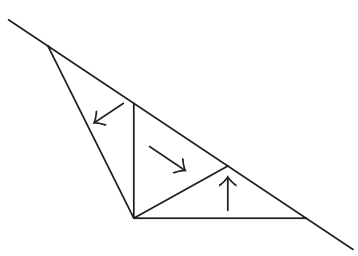

(a)

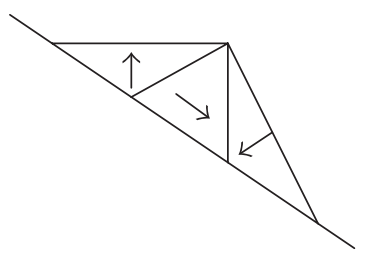

(b)

Figure 2.3

From left to right, the first two arrows represent the gradients

$$
\begin{gathered}
\vec{v}_{1}=3^{-r} \alpha\left(-\frac{1}{2} \sqrt{3} \hat{i}+\frac{1}{2} \hat{j}\right) \\
\vec{v}_{2}=-3^{-r} \alpha \hat{j}
\end{gathered}
$$

respectively. Recall from the introduction that the third and forth arrows represent the gradients

$$
\begin{gathered}
\vec{v}_{3}=3^{-r} \beta \hat{i} \\
\vec{v}_{4}=3^{-r} \beta\left(-\frac{1}{2} \hat{i}+\frac{1}{2} \sqrt{3} \hat{j}\right),
\end{gathered}
$$

respectively. The area of the second triangle is $(1 / 4) 3^{2 r-1 / 2}$, while the area of each of the other three triangles is $(1 / 8) 3^{2 r-1 / 2}$, so this contribution to the Sobolev inner product is 


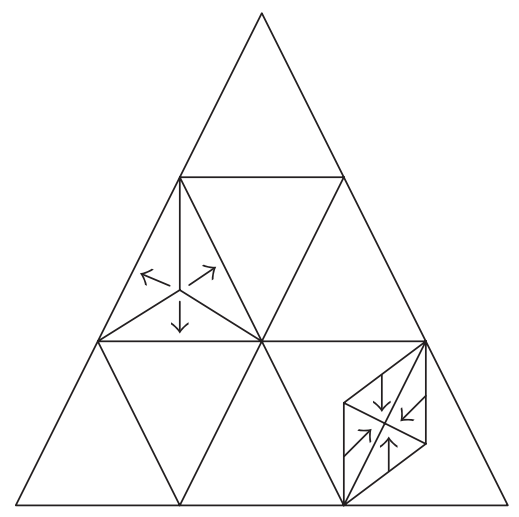

Figure 2.4

just

$$
\begin{gathered}
\frac{1}{8} 3^{2 r-1 / 2} \vec{v}_{1} \cdot \vec{v}+\frac{1}{4} 3^{2 r-1 / 2} \vec{v}_{2} \cdot \vec{v}+\frac{1}{8} 3^{2 r-1 / 2} \vec{v}_{3} \cdot \vec{v}+\frac{1}{8} 3^{2 r-1 / 2} \vec{v}_{4} \cdot \vec{v} \\
=\frac{1}{8} 3^{2 r-1 / 2} \vec{v} \cdot\left(\vec{v}_{1}+2 \vec{v}_{2}+\vec{v}_{3}+\vec{v}_{4}\right) .
\end{gathered}
$$

On the other hand,

$$
\vec{v}_{1}+2 \vec{v}_{2}+\vec{v}_{3}+\vec{v}_{4}=\frac{1}{2} 3^{-r}(\beta-\alpha \sqrt{3}) \hat{i}+\frac{1}{2} 3^{-r}(\beta \sqrt{3}-3 \alpha) \hat{j}
$$

so the contribution vanishes if we set $\beta=\alpha \sqrt{3}$ in our definition of the wavelet. (As we have already pointed out in the introduction, the normalization of the wavelet imposes the additional condition

$$
3\left(\frac{1}{4} 3^{2 r-1 / 2}\right)\left(3^{-r} \alpha\right)^{2}+4\left(\frac{1}{8} 3^{2 r-1 / 2}\right)\left(3^{-r} \beta\right)^{2}=1
$$

which reduces to

$$
\frac{1}{4} \sqrt{3} \alpha^{2}+\frac{1}{2 \sqrt{3}} \beta^{2}=1
$$

so $\beta=\alpha \sqrt{3}$ implies $\alpha=3^{-3 / 4}$ 2.) With this relation between $\alpha$ and $\beta$, the same reasoning shows that the other contribution vanishes as well. This completes the proof of the following orthogonality property.

Orthogonality Property 2.2. If the scales of two Set wavelets differ by a scale factor greater than or equal to $3^{2}$, then the wavelets are orthogonal.

In the introduction we explained why any two Set wavelets with the same scale are orthogonal. The case of successive scales is the only one remaining-and the case where orthogonality partially breaks down. Without loss, we will consider inner products between 0 -level and 1-level wavelets. Clearly, they are orthogonal unless the support triangle of 


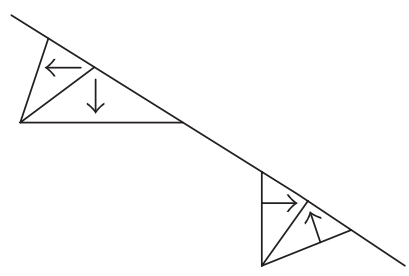

(a)

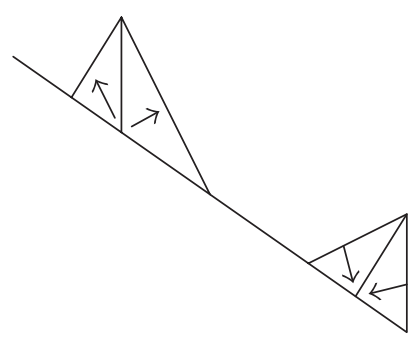

(b)

Figure 2.5

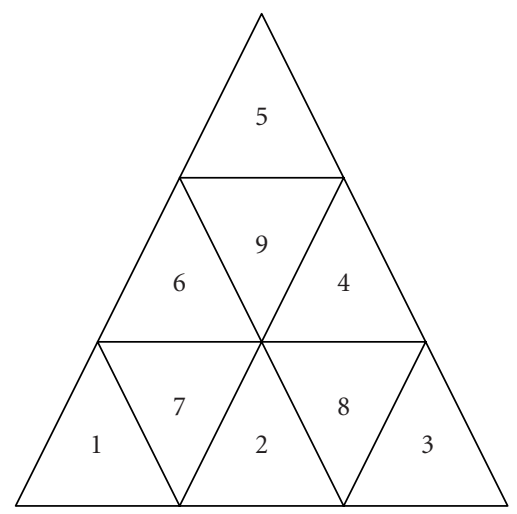

Figure 2.6

the 0-level wavelet is contained in the support triangle of the 1-level wavelet. We may assume that the latter is a + triangle, as our calculations for a - triangle will be identical. The support triangle of the 0 -level wavelet is one of the nine equilateral triangles comprising this + triangle, as illustrated by the diagram in Figure 2.6. For openers, suppose both wavelets are of the first kind. Then the three possibilities for the 1-level wavelet are given by the diagram in Figure 2.7. Now, if the support triangle of the 0-level wavelet is triangle 2,4 , or 6 , then its orthogonality to every possible 1-level wavelet is obvious. Suppose the support triangle is one of the other six-say triangle 7. The possibilities for the 0-level wavelet are given by the diagram in Figure 2.8. To that same triangle, the contribution of the southwest 1-level wavelet is given by the diagram in Figure 2.9. The Sobolev inner product of this basic function with, say, the northwest 0-level wavelet in Figure 2.8 has the decomposition given by the diagrams in Figures 2.10(a) and 2.10(b), but each contribution vanishes by the same calculation that was applied to Figure 2.3. Thus, 0-level Set wavelets of the first kind are orthogonal to 1-level Set wavelets of the first kind. 


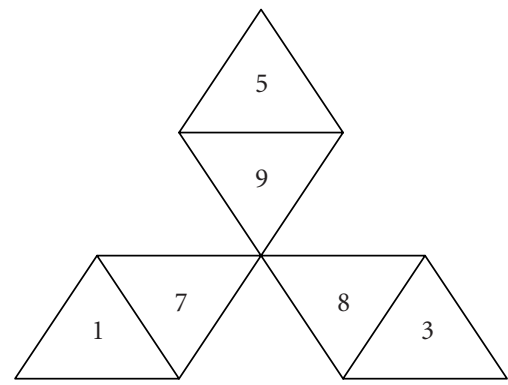

Figure 2.7

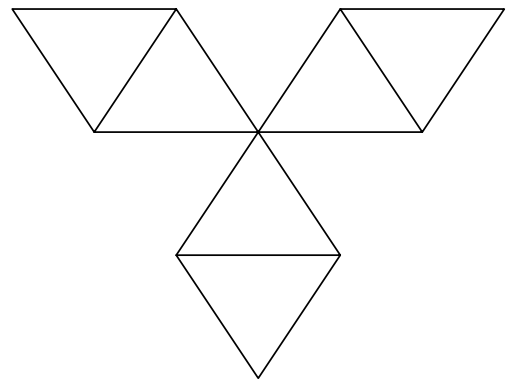

Figure 2.8

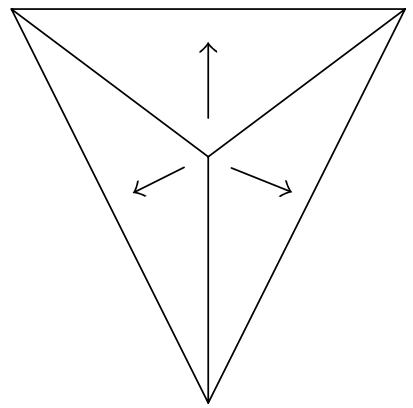

Figure 2.9

Now suppose the 1-level wavelet is of the second kind while the 0-level wavelet is still of the first kind. Then the three possibilities for the 1-level wavelet are given by the diagram in Figure 2.11, with rhombus 1-7 paired with triangle 4, rhombus 3-8 paired with triangle 6 , and rhombus 5-9 paired with triangle 2 . If the support triangle of the 0-level wavelet is triangle 2, 4, or 6, then the case is the same as that represented by Figure 2.10, so we have orthogonality. Suppose the support triangle is one of the other six-again, choosing triangle 7 . The possibilities for the 0 -level wavelet are given by Figure 2.8, but to that same triangle, the contribution of the southwest 1-level wavelet is now given by 


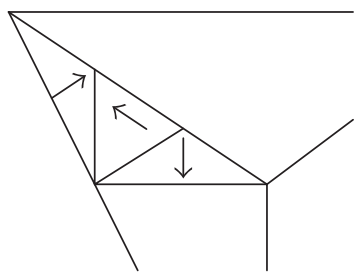

(a)

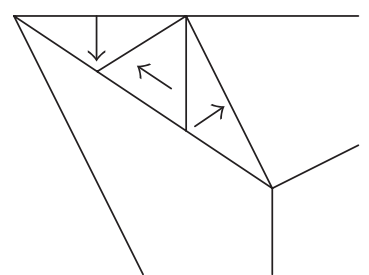

(b)

Figure 2.10

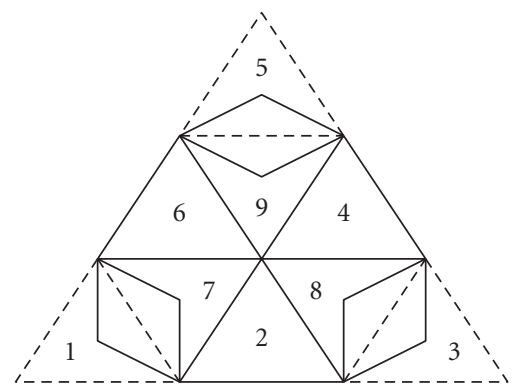

Figure 2.11

the diagram in Figure 2.12. The Sobolev inner product of this function with the northeast 0 -level wavelet in Figure 2.8 is obviously zero. Its inner product with the south 0 -level wavelet is given by the single contribution represented by the diagram in Figure 2.13, while its inner product with the northwest 0-level wavelet is given by the single contribution represented by the diagram in Figure 2.14, but each contribution vanishesonce again-by the same calculation we applied to Figure 2.3. We have now established the following orthogonality property.

Orthogonality Property 2.3. Every 1-level Set wavelet is orthogonal to every 0-level Set wavelet of the first kind.

We may now assume that the 0-level Set wavelet $\Psi$ is of the second kind. We may also assume without loss that the support triangle of the 1-level wavelet is the + triangle with southwest vertex at the origin. Suppose the 1-level Set wavelet $\Psi_{1}^{\iota^{\prime}+}$ is of the first kind-that is, that $\iota^{\prime}=1,2,3$. Then the three possibilities for $\Psi_{1}^{\iota^{\prime}+}$ are given by Figure 2.7. Orthogonality is obvious in case the support triangle of $\Psi$ is triangle 2 , 4, or 6 , so assume that it is one of the other six-once again, choosing triangle 7 . The possibilities for the 0-level wavelet $\Psi$ are given by the diagrams in Figures 2.15(a), 2.15(b), and 2.15(c). The contribution of the southwest 1-level wavelet to that same triangle is given by Figure 2.9, and the Sobolev inner product of this basic function with, say, the northwest 


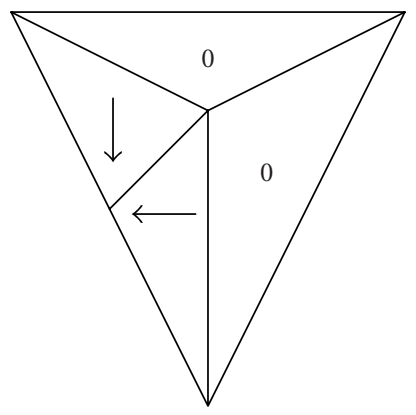

Figure 2.12

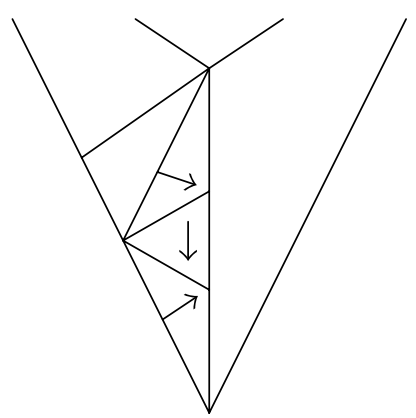

Figure 2.13

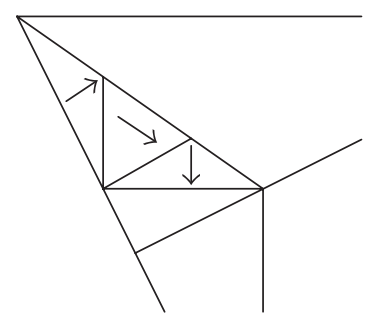

Figure 2.14

0-level wavelet (given by Figure 2.15(c)) is represented by the diagram in Figure 2.16, where we calculate the dot product of each vector with the vector in Figure 2.9 whose triangle includes the triangle of the given vector. Now, by Orthogonality Property 2.1, the basic function associated with the equilateral triangle in Figure 2.16 does not contribute to the inner product. The contribution arising from the basic function associated with the rhombus in Figure 2.16 has a decomposition represented by the diagrams in Figures 2.17(a) and 2.17(b). These two contributions to the inner product are equal by symmetry, 


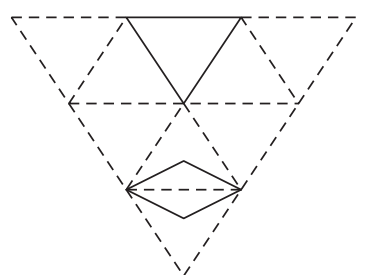

(a)

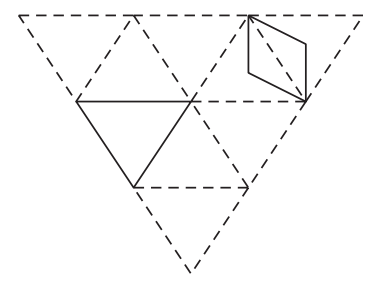

(b)

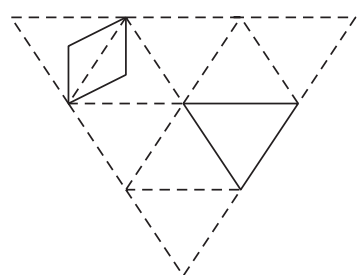

(c)

Figure 2.15

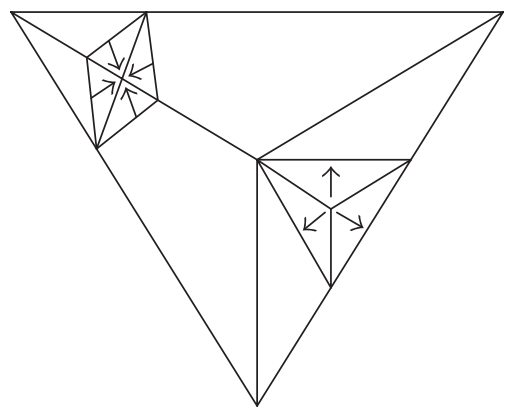

Figure 2.16

so we consider Figure 2.17(a) only. The 1-level gradient is just

$$
\vec{v}=3^{-5 / 4} \sqrt{2}\left(\frac{1}{2} \sqrt{3} \hat{i}+\frac{1}{2} \hat{j}\right)
$$

while the 0-level gradients in the left and right triangles are just

$$
\begin{gathered}
\vec{v}_{L}=\beta \hat{i}, \\
\vec{v}_{R}=\beta\left(-\frac{1}{2} \hat{i}+\frac{1}{2} \sqrt{3} \hat{j}\right),
\end{gathered}
$$

respectively. Since $\vec{v}_{R} \cdot \vec{v}=0$ and the area of the left triangle is $(1 / 24) \sqrt{3}$, the Sobolev inner product is therefore given by

$$
\int \nabla \Psi \cdot \nabla \Psi_{1}^{1+}=2\left(\frac{1}{24} \sqrt{3} \vec{v}_{L} \cdot \vec{v}\right)=\frac{1}{24} 3^{-1 / 4} \sqrt{2} \beta .
$$

Since we have already determined that $\beta=3^{-1 / 4} 2$, it follows that

$$
\int \nabla \Psi \cdot \nabla \Psi_{1}^{1+}=\frac{1}{6 \sqrt{6}}
$$




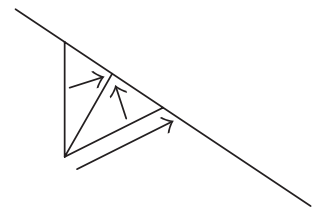

(a)

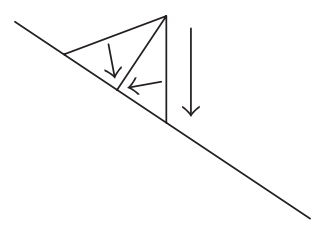

(b)

Figure 2.17

in this case-that is, where $\Psi$ is the northwest wavelet in triangle 7 . By the symmetry property of Figure 2.9, the inner product is the same in the cases where $\Psi$ is the northeast wavelet or the south wavelet in triangle 7 . Thus

$$
\int \nabla \Psi_{07}^{\iota} \cdot \nabla \Psi_{1}^{1+}=\frac{1}{6 \sqrt{6}}, \quad \iota=4,5,6,
$$

where we adopt the special notation described in the introduction. For the southeast (resp., north) 1-level wavelet $\Psi_{1}^{2+}$ (resp., $\Psi_{1}^{3+}$ ) we obviously have

$$
\begin{gathered}
\int \nabla \Psi_{07}^{\iota} \cdot \nabla \Psi_{1}^{2+}=0, \\
\text { (resp., } \left.\int \nabla \Psi_{07}^{\iota} \cdot \nabla \Psi_{1}^{3+}=0\right)
\end{gathered}
$$

for $\iota=4,5,6$ because triangle 7 is disjoint from intsupp $\Psi_{1}^{2+}$ (resp., intsupp $\Psi_{1}^{3+}$ ). The same reduction argument and calculation yield the other inner products:

$$
\begin{aligned}
& \int \nabla \Psi_{08}^{\iota} \cdot \nabla \Psi_{1}^{1+}=0, \\
& \int \nabla \Psi_{08}^{\iota} \cdot \nabla \Psi_{1}^{2+}=\frac{1}{6 \sqrt{6}}, \\
& \int \nabla \Psi_{08}^{\iota} \cdot \nabla \Psi_{1}^{3+}=0, \\
& \int \nabla \Psi_{09}^{\iota} \cdot \nabla \Psi_{1}^{1+}=0, \\
& \int \nabla \Psi_{09}^{\iota} \cdot \nabla \Psi_{1}^{2+}=0, \\
& \int \nabla \Psi_{09}^{\iota} \cdot \nabla \Psi_{1}^{3+}=\frac{1}{6 \sqrt{6}}, \\
& \int \nabla \Psi_{01}^{\iota} \cdot \nabla \Psi_{1}^{1+}=-\frac{1}{6 \sqrt{6}}, \\
& \int \nabla \Psi_{01}^{\iota} \cdot \nabla \Psi_{1}^{2+}=0, \\
& \int \nabla \Psi_{01}^{\iota} \cdot \nabla \Psi_{1}^{3+}=0,
\end{aligned}
$$




$$
\begin{aligned}
& \int \nabla \Psi_{03}^{\iota} \cdot \nabla \Psi_{1}^{1+}=0 \\
& \int \nabla \Psi_{03}^{\iota} \cdot \nabla \Psi_{1}^{2+}=-\frac{1}{6 \sqrt{6}}, \\
& \int \nabla \Psi_{03}^{\iota} \cdot \nabla \Psi_{1}^{3+}=0 \\
& \int \nabla \Psi_{05}^{\iota} \cdot \nabla \Psi_{1}^{1+}=0, \\
& \int \nabla \Psi_{05}^{\iota} \cdot \nabla \Psi_{1}^{2+}=0, \\
& \int \nabla \Psi_{05}^{\iota} \cdot \nabla \Psi_{1}^{3+}=-\frac{1}{6 \sqrt{6}}
\end{aligned}
$$

for $\iota=4,5,6$. We have already pointed out that

$$
\int \nabla \Psi_{02}^{\iota} \cdot \nabla \Psi_{1}^{\iota^{\prime}+}=\int \nabla \Psi_{04}^{\iota} \cdot \nabla \Psi_{1}^{\iota^{\prime}+}=\int \nabla \Psi_{06}^{\prime} \cdot \nabla \Psi_{1}^{\iota^{\prime}+}=0
$$

for $\iota=4,5,6$ and $\iota^{\prime}=1,2,3$.

Having calculated the inner product of $\Psi$ with $\Psi_{1}^{\iota^{\prime}+}$ for every case where the 1-level wavelet is of the first kind - that is, where $\iota^{\prime}=1,2,3$ - we now turn to the final set of cases, where the 1 -level wavelet is of the second kind-that is, where $\iota^{\prime}=4,5,6$. The three possibilities for $\Psi_{1}^{\iota^{\prime}+}$ are now given by Figure 2.11. Suppose the support triangle of $\Psi$ is triangle 2. The possibilities for the 0-level wavelet $\Psi$ are given by the diagrams in Figures 2.18(a), 2.18 (b), and 2.18(c). The contribution of the north 1-level wavelet $\psi_{1}^{4+}$ to that same triangle is given by the diagram in Figure 2.19. This is the same situation reflected by our comparison of Figure 2.15 with Figure 2.9. Hence

$$
\int \nabla \Psi \cdot \nabla \Psi_{1}^{4+}=\frac{1}{6 \sqrt{6}}
$$

in each of the three cases for $\Psi$ given by Figure 2.18 in triangle 2. Thus

$$
\int \nabla \Psi_{02}^{\iota} \cdot \nabla \Psi_{1}^{4+}=\frac{1}{6 \sqrt{6}}, \quad \iota=4,5,6
$$

Since triangle 2 is disjoint from int $\operatorname{supp} \Psi_{1}^{5+}$ and int $\operatorname{supp} \Psi_{1}^{6+}$, we also have

$$
\begin{aligned}
& \int \nabla \Psi_{02}^{\iota} \cdot \nabla \Psi_{1}^{5+}=0 \\
& \int \nabla \Psi_{02}^{\iota} \cdot \nabla \Psi_{1}^{6+}=0
\end{aligned}
$$




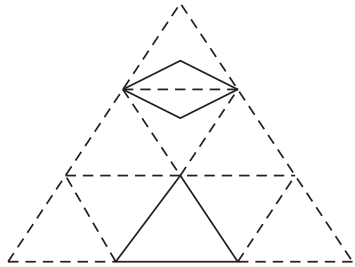

(a)

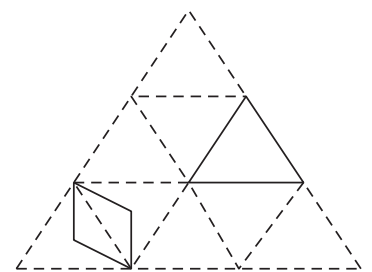

(b)

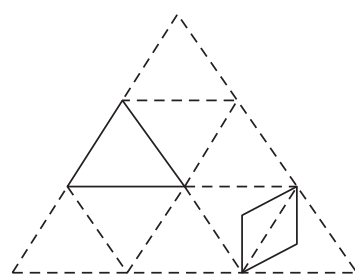

(c)

Figure 2.18

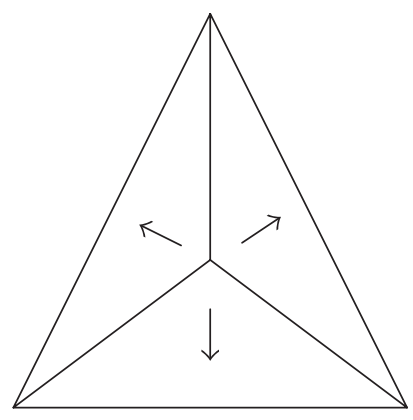

Figure 2.19

for $\iota=4,5,6$. The situation is similar in the cases where the support triangle of the given 0 -level wavelet is triangle 4 or triangle 6 . Indeed,

$$
\begin{aligned}
& \int \nabla \Psi_{04}^{\iota} \cdot \nabla \Psi_{1}^{4+}=0, \\
& \int \nabla \Psi_{04}^{\iota} \cdot \nabla \Psi_{1}^{5+}=\frac{1}{6 \sqrt{6}}, \\
& \int \nabla \Psi_{04}^{\iota} \cdot \nabla \Psi_{1}^{6+}=0, \\
& \int \nabla \Psi_{06}^{\iota} \cdot \nabla \Psi_{1}^{4+}=0, \\
& \int \nabla \Psi_{06}^{\iota} \cdot \nabla \Psi_{1}^{5+}=0, \\
& \int \nabla \Psi_{06}^{\iota} \cdot \nabla \Psi_{1}^{6+}=\frac{1}{6 \sqrt{6}}
\end{aligned}
$$

for $\iota=4,5,6$.

Now suppose the support triangle of the 0-level wavelet $\Psi$ is triangle 7 in Figure 2.11. The possibilities for $\Psi$ are given by Figure 2.15, while the contribution of the southwest 1-level wavelet $\Psi_{1}^{5+}$ is given by Figure 2.12. (Neither $\Psi_{1}^{6+}$ nor $\Psi_{1}^{4+}$ contributes anything to 


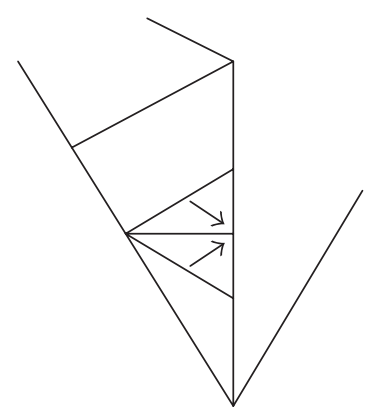

Figure 2.20

this triangle.) Consider the Sobolev inner product of the south 0 -level wavelet $\Psi=\Psi_{07}^{4}$ with $\Psi_{1}^{5+}$, which is represented by the diagram in Figure 2.20. The gradients in the small upper and lower triangles are just

$$
\vec{v}_{ \pm}=\beta\left(\frac{1}{2} \hat{i} \mp \frac{1}{2} \sqrt{3} \hat{j}\right)
$$

respectively, while the gradient of $\Psi_{1}^{5+}$ in the triangle including them is just

$$
\vec{v}=-\frac{1}{3} \beta \hat{i}
$$

Since the area of each small triangle is $(1 / 24) \sqrt{3}$, it follows that

$$
\int \nabla \Psi_{07}^{4} \cdot \nabla \Psi_{1}^{5+}=\sum_{ \pm} \vec{v}_{ \pm} \cdot \vec{v}\left(\frac{1}{24} \sqrt{3}\right)=-\frac{1}{72} \sqrt{3} \beta^{2}=-\frac{1}{18}
$$

For the northeast 0-level wavelet $\Psi=\Psi_{07}^{5}$, the Sobolev inner product with $\Psi_{1}^{5+}$ has the decomposition into contributions represented by the diagrams in Figures 2.21(a) and 2.21(b). Consider the contribution given by the first diagram. The gradients in the small upper and lower triangles are just

$$
\begin{gathered}
\vec{v}_{+}=\alpha \hat{j}, \\
\vec{v}_{-}=\alpha\left(-\frac{1}{2} \sqrt{3} \hat{i}-\frac{1}{2} \hat{j}\right),
\end{gathered}
$$

respectively, while the gradient of $\Psi_{1}^{5+}$ in the triangle including them is just

$$
\vec{v}=\frac{1}{3} \beta\left(-\frac{1}{2} \hat{i}-\frac{1}{2} \sqrt{3} \hat{j}\right)
$$


The areas of the upper and lower triangles are $(1 / 12) \sqrt{3}$ and $(1 / 24) \sqrt{3}$, respectively, so this contribution has the value

$$
\begin{aligned}
\frac{1}{12} \sqrt{3} \vec{v} \cdot \vec{v}_{+}+\frac{1}{24} \sqrt{3} \vec{v} \cdot \vec{v}_{-} & =\frac{1}{12} \sqrt{3}\left(-\frac{1}{6} \sqrt{3} \alpha \beta\right)+\frac{1}{24} \sqrt{3}\left(\frac{1}{6} \sqrt{3} \alpha \beta\right) \\
& =-\frac{1}{48} \alpha \beta=-\frac{1}{36} .
\end{aligned}
$$

By symmetry, the other contribution has the same value. Hence

$$
\int \nabla \Psi_{07}^{5} \cdot \nabla \Psi_{1}^{5+}=-\frac{1}{18} .
$$

For the northwest 0-level wavelet $\Psi=\Psi_{07}^{6}$, the Sobolev inner product with $\Psi_{1}^{5+}$ is represented by the diagram in Figure 2.22. By symmetry, this inner product is equal to the inner product represented by Figure 2.20. Thus

$$
\int \nabla \Psi_{07}^{6} \cdot \nabla \Psi_{1}^{5+}=-\frac{1}{18} .
$$

As we have already mentioned, $\Psi_{1}^{4+}$ and $\Psi_{1}^{6+}$ contribute nothing to triangle 7 , so

$$
\int \nabla \Psi_{07}^{\iota} \cdot \nabla \Psi_{1}^{6+}=\int \nabla \Psi_{07}^{\iota} \cdot \nabla \Psi_{1}^{4+}=0, \quad \iota=4,5,6 .
$$

All remaining cases are identical to the cases we have discussed, so we are now in a position to evaluate all remaining inner products:

$$
\begin{aligned}
& \int \nabla \Psi_{08}^{\iota} \cdot \nabla \Psi_{1}^{6+}=-\frac{1}{18} \\
& \int \nabla \Psi_{08}^{\iota} \cdot \nabla \Psi_{1}^{4+}=\int \nabla \Psi_{08}^{\iota} \cdot \nabla \Psi_{1}^{5+}=0 \\
& \int \nabla \Psi_{09}^{\iota} \cdot \nabla \Psi_{1}^{4+}=-\frac{1}{18}, \\
& \int \nabla \Psi_{09}^{\iota} \cdot \nabla \Psi_{1}^{5+}=\int \nabla \Psi_{09}^{\iota} \cdot \nabla \Psi_{1}^{6+}=0 \\
& \int \nabla \Psi_{01}^{\iota} \cdot \nabla \Psi_{1}^{5+}=-\frac{1}{18}, \\
& \int \nabla \Psi_{01}^{\iota} \cdot \nabla \Psi_{1}^{6+}=\int \nabla \Psi_{01}^{\iota} \cdot \nabla \Psi_{1}^{4+}=0 \\
& \int \nabla \Psi_{03}^{\iota} \cdot \nabla \Psi_{1}^{6+}=-\frac{1}{18}, \\
& \int \nabla \Psi_{03}^{\iota} \cdot \nabla \Psi_{1}^{4+}=\int \nabla \Psi_{03}^{\iota} \cdot \nabla \Psi_{1}^{5+}=0 \\
& \int \nabla \Psi_{05}^{\iota} \cdot \nabla \Psi_{1}^{4+}=-\frac{1}{18}, \\
& \int \nabla \Psi_{05}^{\iota} \cdot \nabla \Psi_{1}^{5+}=\int \nabla \Psi_{05}^{\iota} \cdot \nabla \Psi_{1}^{6+}=0
\end{aligned}
$$

for $\iota=4,5,6$. 


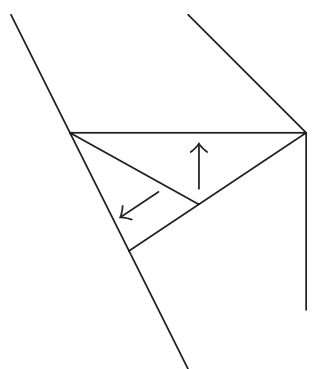

(a)

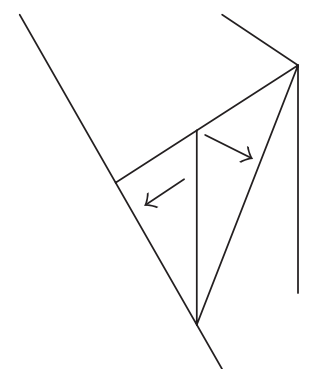

(b)

Figure 2.21

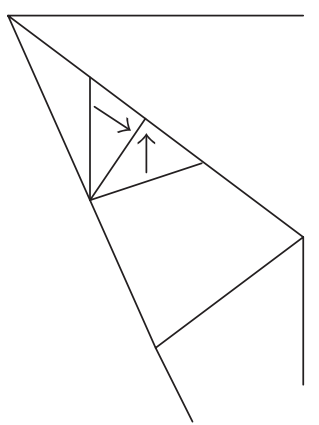

Figure 2.22

\section{The Sobolev overlap matrix}

Following the notation adopted in the introduction, we index our set of wavelets with a support triangle index \pm , a mother wavelet index $\iota$, a discrete translation parameter $\nu \in \mathbb{Z}^{2}$, and a discrete scaling parameter $r \in \mathbb{Z}$. The wavelets are given by

$$
\Psi_{r \nu}^{\prime \pm}(\vec{x})=\Psi^{\prime \pm}\left(3^{-r} \vec{x}-3 v_{0} \hat{i}-3 v_{1}\left(\frac{1}{2} \hat{i}+\frac{1}{2} \sqrt{3} \hat{j}\right)\right)
$$

The scaling of the amplitude needed for the normalization

$$
\int\left|\nabla \Psi_{r v}^{\prime \pm}\right|^{2}=1
$$

is unity in two dimensions. The overlap matrix is given by

$$
S_{r r^{\prime} ; \nu v^{\prime} ; l l^{\prime} ; \pm \pm^{\prime}}=\int \nabla \Psi_{r \nu}^{l \pm} \cdot \nabla \Psi_{r^{\prime} \nu^{\prime}}^{l^{\prime} \pm^{\prime}},
$$


and obviously it has the property

$$
S_{r+s, r^{\prime}+s ; v v^{\prime} ; l^{\prime} ; \pm \pm \pm^{\prime}}=S_{r r^{\prime} ; \nu v^{\prime} ; l^{\prime} ; \pm \pm^{\prime}}
$$

so it has the form

$$
S_{r r^{\prime} ; \nu v^{\prime} ; u l^{\prime} ; \pm \pm \pm^{\prime}}=T_{r-r^{\prime} ; \nu v^{\prime} ; l l^{\prime} ; \pm \pm \pm^{\prime}} .
$$

This means that if we consider the Fourier series

$$
\widehat{T}_{\nu \nu^{\prime} ; l^{\prime} ; \pm \pm^{\prime}}(\omega)=\sum_{s=-\infty}^{\infty} T_{s ; \nu v^{\prime} ; l^{\prime} ; \pm \pm^{\prime}} e^{i s \omega},
$$

a positive lower bound on the manifestly positive matrix $S$ would follow from a positive, $\omega$-independent lower bound on this positive matrix-valued function of $\omega$. Now the mutual orthogonality of wavelets on the same scale immediately implies

$$
T_{0}=1
$$

Moreover, the orthogonality between $r$-level wavelets and $r^{\prime}$-level wavelets verified in the previous section for $\left|r-r^{\prime}\right|>1$ imposes the condition

$$
T_{s}=0, \quad s \neq 0, \pm 1 .
$$

It is obvious that

$$
T_{-1}=T_{1}^{*}
$$

so our matrix-valued function of $\omega$ reduces to

$$
\widehat{T}(\omega)=1+T_{1} e^{i \omega}+T_{1}^{*} e^{-i \omega}
$$

The desired lower bound on $\widehat{T}(\omega)$ would certainly follow from

$$
\left\|T_{1} e^{i \omega}+T_{1}^{*} e^{-i \omega}\right\| \leq c<1,
$$

and the proof of such a bound is the goal of this section and the next.

Our next step is to eliminate the discrete translation parameter from the problem. By the support properties of the wavelets, we have

$$
T_{1 ; \nu v^{\prime} ; l^{\prime} ; \pm \pm^{\prime}}=0, \quad v^{\prime}-3 v \notin\{0,1,2\}^{2} .
$$

On the other hand, the formula

$$
T_{1 ; \nu v^{\prime} ; l^{\prime} ; \pm \pm^{\prime}}=\int \nabla \Psi_{1 v}^{\prime \pm} \cdot \nabla \Psi_{0 \nu^{\prime}}^{i^{\prime} \pm^{\prime}}
$$

together with the translation-covariance property of the wavelets, implies

$$
T_{1 ; \nu+\mu, v^{\prime}+3 \mu ; l^{\prime} ; \pm \pm \pm^{\prime}}=T_{1 ; \nu v^{\prime} ; l^{\prime} ; \pm \pm \pm^{\prime}} .
$$


Hence

$$
T_{1 ; \nu, 3 \mu+\sigma ; l^{\prime} ; \pm \pm^{\prime}}=T_{1 ; 00, \sigma ; u^{\prime} ; \pm \pm^{\prime}} \delta_{\mu \nu}, \quad \sigma \in\{0,1,2\}^{2},
$$

and for convenience we decompose this residual matrix into nine $12 \times 12$ submatrices:

$$
Q_{u^{\prime} ; \pm \pm^{\prime}}^{\sigma}=T_{1 ; 00, \sigma ; l^{\prime} ; \pm \pm^{\prime}}, \quad \sigma \in\{0,1,2\}^{2}
$$

The point is that

$$
\left\|T_{1}\right\|^{2}=\left\|\left\{T_{1 ; 00, \sigma ; l^{\prime} ; \pm \pm^{\prime}}\right\}\right\|^{2} \leq \sum_{\sigma \in\{0,1,2\}^{2}}\left\|Q^{\sigma}\right\|^{2}
$$

Indeed, for each $\sigma$ we have the $12 \times 12$ matrix equation

$$
\begin{aligned}
\left(T_{1}^{N}\right)_{\nu, 3 \mu+\sigma} & =\sum_{\nu^{\prime}, \nu^{\prime \prime}, \ldots, \nu^{(N-1)}} T_{1 ; \nu \nu^{\prime}} T_{1 ; \nu^{\prime} \nu^{\prime \prime}} \cdots T_{1 ; \nu^{(N-1)}, 3 \mu+\sigma} \\
& =\sum_{\mu^{\prime}, \mu^{\prime \prime}, \ldots, \mu^{(N-1)}} \sum_{\sigma^{\prime}, \sigma^{\prime \prime}, \ldots, \sigma^{(N-1)}} Q^{\sigma^{\prime}} Q^{\sigma^{\prime \prime}} \cdots Q^{\sigma^{(N-1)}} Q^{\sigma} \delta_{\mu^{\prime} \nu} \delta_{\mu^{\prime \prime}, 3 \mu^{\prime}+\sigma^{\prime}} \cdots \delta_{\mu, 3 \mu^{(N-1)}+\sigma^{(N-1)}} \\
& =\sum_{\sigma^{\prime}, \sigma^{\prime \prime}, \ldots, \sigma^{(N-1)}} Q^{\sigma^{\prime}} Q^{\sigma^{\prime \prime}} \cdots Q^{\sigma^{(N-1)}} Q^{\sigma} \delta_{\mu, 3^{N-1} \nu+3^{N-2} \sigma^{\prime}+\cdots+3 \sigma^{(N-2)}+\sigma^{(N-1)}}
\end{aligned}
$$

It follows from the triadic representation

$$
\mu=3^{N-1} v_{\mu}+3^{N-2} \sigma_{\mu}^{\prime}+\cdots+3 \sigma_{\mu}^{(N-2)}+\sigma_{\mu}^{(N-1)}
$$

that

$$
\delta_{\mu, 3^{N-1} \nu+3^{N-2} \sigma^{\prime}+\cdots+3 \sigma^{(N-2)}+\sigma^{(N-1)}}=\delta_{\nu_{\mu}} \prod_{k=1}^{N-1} \delta_{\sigma_{\mu}^{(k)} \sigma^{(k)}},
$$

and so

$$
\left(T_{1}^{N}\right)_{\nu, 3 \mu+\sigma}=\delta_{\gamma_{\mu} \nu} Q^{\sigma_{\mu}^{\prime}} Q^{\sigma_{\mu}^{\prime \prime}} \cdots Q^{\sigma_{\mu}^{(N-1)}} Q^{\sigma}
$$

For an arbitrary square-summable 12-vector-valued sequence $\left(\gamma_{\nu} \mid \nu \in \mathbb{Z}\right)$, this yields

$$
\left(T_{1}^{N} \gamma\right)_{\nu}=\sum_{\sigma^{\prime}, \sigma^{\prime \prime}, \ldots, \sigma^{(N-1)}, \sigma} Q^{\sigma^{\prime}} Q^{\sigma^{\prime \prime}} \cdots Q^{\sigma^{(N-1)}} Q^{\sigma} \gamma_{3^{N} \nu+3^{N-1} \sigma^{\prime}+\cdots+3 \sigma^{(N-1)}+\sigma}
$$

from which we obtain the generalization

$$
\left\|T_{1}^{N}\right\|^{2} \leq \sum_{\sigma^{\prime}, \sigma^{\prime \prime}, \ldots, \sigma^{(N-1)}, \sigma}\left\|Q^{\sigma^{\prime}} Q^{\sigma^{\prime \prime}} \cdots Q^{\sigma^{(N-1)}} Q^{\sigma}\right\|^{2}
$$

of (3.17). This estimation lies at the heart of proving (3.11) with a refinement of the constant $c$. 
We now use another support property of the wavelet system to deduce that certain $6 \times 6$ submatrices vanish. Indeed,

$$
\begin{array}{ll}
Q_{++}^{\sigma}=0, & \sigma=(2,1),(1,2),(2,2), \\
Q_{+-}^{\sigma}=0, & \sigma=(0,2),(1,2),(2,2),(1,1),(2,1),(2,0), \\
Q_{-+}^{\sigma}=0, & \sigma=(0,0),(0,1),(1,0),(1,1),(2,0),(0,2), \\
Q_{--}^{\sigma}=0, & \sigma=(0,0),(0,1),(1,0),
\end{array}
$$

can be inferred from the diagrams in Figure 3.1, where the small equilateral triangles each support six unit-scale wavelets (so the side of each small triangle is 3 units long) and the large equilateral triangles each support six 3-scale wavelets. Moreover, isotropy implies

$$
\begin{aligned}
& Q_{+ \pm}^{00}=Q_{-\mp}^{22}, \\
& Q_{+ \pm}^{01}=Q_{-\mp}^{21}, \\
& Q_{+ \pm}^{10}=Q_{-\mp}^{12}, \\
& Q_{++}^{02}=Q_{--}^{20}, \\
& Q_{++}^{11}=Q_{--}^{11}, \\
& Q_{++}^{20}=Q_{--}^{02} .
\end{aligned}
$$

If we now reindex the small triangles in the large + triangle in Figure 1.9(a), then these relations naturally dictate the reindexing of the small triangles in the large - triangle, chosen in Figure 1.9(b). The point is that there are only nine distinct $6 \times 6$ matricesone for each small triangle in either larger triangle. Let $A^{(j)}$ denote the matrix associated with the $j$ th small triangle. Thus

$$
\begin{aligned}
& A^{(1)}=(3.25 .+), \\
& A^{(2)}=(3.27 .+), \\
& A^{(3)}=(3.30), \\
& A^{(4)}=(3.29), \\
& A^{(5)}=(3.28), \\
& A^{(6)}=(3.26 .-), \\
& A^{(7)}=(3.25 .-), \\
& A^{(8)}=(3.27 .-), \\
& A^{(9)}=(3.26 .-) .
\end{aligned}
$$


522 Osiris wavelets and Set wavelets

On the other hand, we have the $6 \times 6$ matrix equation

$$
\begin{aligned}
& \left(Q^{\sigma^{\prime}} Q^{\sigma^{\prime \prime}} \cdots Q^{\sigma^{(N-1)}} Q^{\sigma^{(N)}}\right)_{ \pm \pm(N)}
\end{aligned}
$$

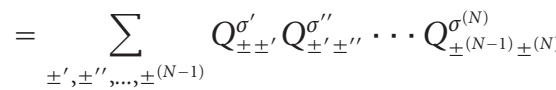

$$
\begin{aligned}
& =\left[\delta_{ \pm, \sigma^{\prime}\left( \pm^{\prime}\right)} Q_{ \pm \pm^{\prime}}^{\sigma^{\prime}} Q_{ \pm^{\prime} \pm^{\prime \prime}}^{\sigma^{\prime \prime}} \cdots Q_{ \pm}^{\sigma^{(N)}(N)} \pm^{(N)}\right]_{ \pm}^{(k)=\sigma^{(k+1)}\left( \pm^{(k+1)}\right), k=1, \ldots, N-1},
\end{aligned}
$$

where $\sigma( \pm)$ is the unique value of $\hat{ \pm}$ such that $Q_{\hat{x}, \pm}^{\sigma} \neq 0$. Thus

$$
\begin{aligned}
& (0,0)( \pm)=(1,0)( \pm)=(0,1)( \pm)=+, \\
& (1,1)( \pm)=(2,0)( \pm)=(0,2)( \pm)= \pm, \\
& (2,1)( \pm)=(1,2)( \pm)=(2,2)( \pm)=-
\end{aligned}
$$

are implied by (3.24). The bound (3.23) is reduced to

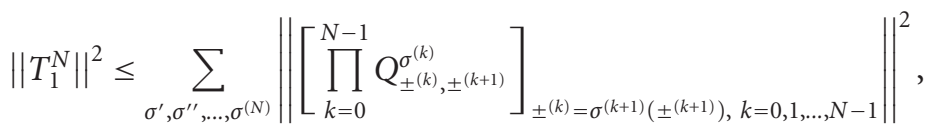

but since

$$
\begin{aligned}
& Q_{\sigma(+),+}^{\sigma}= \begin{cases}A^{(1)}, & \sigma=(0,0), \\
A^{(2)}, & \sigma=(1,0), \\
A^{(6)}, & \sigma=(0,1), \\
A^{(4)}, & \sigma=(1,1), \\
A^{(5)}, & \sigma=(0,2), \\
A^{(8)}, & \sigma=(1,2), \\
A^{(9)}, & \sigma=(2,1), \\
A^{(7)}, & \sigma=(2,2),\end{cases} \\
& Q_{\sigma(-),-}^{\sigma}= \begin{cases}A^{(7)}, & \sigma=(0,0), \\
A^{(8)}, & \sigma=(1,0), \\
A^{(9)}, & \sigma=(0,1), \\
A^{(4)}, & \sigma=(1,1), \\
A^{(3)}, & \sigma=(0,2), \\
A^{(5)}, & \sigma=(2,0), \\
A^{(2)}, & \sigma=(1,2), \\
A^{(6)}, & \sigma=(2,1), \\
A^{(1)}, & \sigma=(2,2),\end{cases}
\end{aligned}
$$



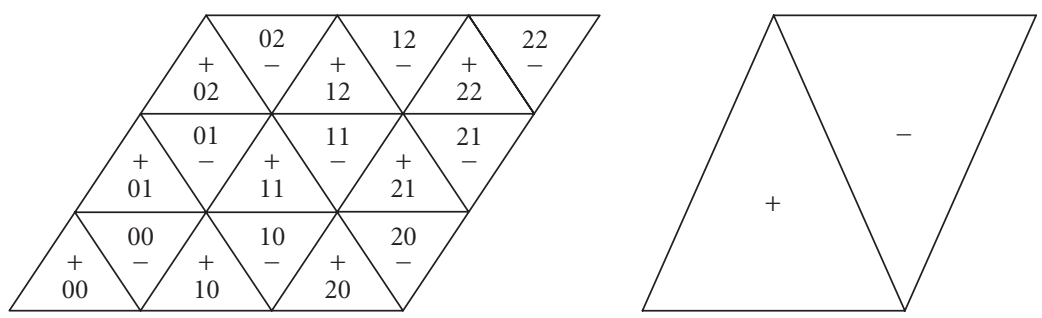

Figure 3.1

we can rewrite this bound as

$$
\left\|T_{1}^{N}\right\|^{2} \leq\left(\prod_{k=1}^{N} \sum_{j_{k}=1}^{9}\right)\left\|A^{\left(j_{1}\right)} A^{\left(j_{2}\right)} \cdots A^{\left(j_{N}\right)}\right\|^{2} .
$$

\section{The nine matrices $A^{(j)}$}

Following the notation adopted for 0-level wavelets and their support triangles in the standard support triangle for 1-level wavelets, we have

$$
A_{u^{\prime}}^{(j)}=\int \nabla \Psi_{0 j}^{\iota} \cdot \nabla \Psi_{1}^{\iota^{\prime}+} .
$$

By Orthogonality Property 2.3, we know that

$$
A_{\iota^{\prime}}^{(j)}=0, \quad \iota=1,2,3,
$$

so we choose to write our $6 \times 6$ matrices in $3 \times 3$ blocks:

$$
A^{(j)}=\left[\begin{array}{cc}
0 & 0 \\
B^{(j)} & C^{(j)}
\end{array}\right] .
$$

Clearly,

$$
\begin{aligned}
A^{(j)} A^{\left(j^{\prime}\right)} & =\left[\begin{array}{cc}
0 & 0 \\
C^{(j)} B^{\left(j^{\prime}\right)} & C^{(j)} C^{\left(j^{\prime}\right)}
\end{array}\right], \\
A^{(j) *} & =\left[\begin{array}{ll}
0 & B^{(j) *} \\
0 & C^{(j) *}
\end{array}\right], \\
A^{(j)} A^{(j) *} & =\left[\begin{array}{lc}
0 & 0 \\
0 & B^{(j)} B^{(j) *}+C^{(j)} C^{(j) *}
\end{array}\right] .
\end{aligned}
$$

In particular,

$$
\left\|A^{(j)}\right\|^{2}=\left\|A^{(j)} A^{(j) *}\right\|=\left\|B^{(j)} B^{(j) *}+C^{(j)} C^{(j) *}\right\| .
$$


The matrices $B^{(j)}$ (resp., $C^{(j)}$ ) involve Sobolev inner products of 0-level Set wavelets of the second kind with 1-level Set wavelets of the first kind (resp., second kind). Considering the case $j=1$, we draw on (2.17) to obtain

$$
B^{(1)}=-\frac{1}{6 \sqrt{6}}\left[\begin{array}{lll}
1 & 0 & 0 \\
1 & 0 & 0 \\
1 & 0 & 0
\end{array}\right]
$$

and on (2.38) and (2.39) to obtain

$$
C^{(1)}=-\frac{1}{18}\left[\begin{array}{lll}
1 & 0 & 0 \\
1 & 0 & 0 \\
1 & 0 & 0
\end{array}\right]
$$

We calculate

$$
B^{(1)} B^{(1) *}+C^{(1)} C^{(1) *}=\frac{5}{648}\left[\begin{array}{lll}
1 & 1 & 1 \\
1 & 1 & 1 \\
1 & 1 & 1
\end{array}\right] .
$$

The eigenvalues of the integer matrix are 0 and 3, so by (4.7) we have

$$
\left\|A^{(1)}\right\|^{2}=\frac{5}{216}
$$

On the other hand, we infer from (2.20) that

$$
B^{(2)}=B^{(4)}=B^{(6)}=0 .
$$

Considering the cases $j=2,4,6$, we draw on (2.22), (2.23), and (2.24) to obtain

$$
\begin{aligned}
& C^{(2)}=\frac{1}{6 \sqrt{6}}\left[\begin{array}{lll}
1 & 0 & 0 \\
1 & 0 & 0 \\
1 & 0 & 0
\end{array}\right], \\
& C^{(4)}=\frac{1}{6 \sqrt{6}}\left[\begin{array}{lll}
0 & 1 & 0 \\
0 & 1 & 0 \\
0 & 1 & 0
\end{array}\right], \\
& C^{(6)}=\frac{1}{6 \sqrt{6}}\left[\begin{array}{lll}
0 & 0 & 1 \\
0 & 0 & 1 \\
0 & 0 & 1
\end{array}\right],
\end{aligned}
$$

respectively. Thus

$$
C^{(2)} C^{(2) *}=C^{(4)} C^{(4) *}=C^{(6)} C^{(6) *}=\frac{1}{216}\left[\begin{array}{lll}
1 & 1 & 1 \\
1 & 1 & 1 \\
1 & 1 & 1
\end{array}\right]
$$


and so by (4.7) we have

$$
\left\|A^{(j)}\right\|^{2}=\left\|C^{(j)} C^{(j) *}\right\|=\frac{1}{72}, \quad j=2,4,6 .
$$

Considering the case $j=3$, we draw on (2.18) to obtain

$$
B^{(3)}=-\frac{1}{6 \sqrt{6}}\left[\begin{array}{lll}
0 & 1 & 0 \\
0 & 1 & 0 \\
0 & 1 & 0
\end{array}\right],
$$

and on (2.40) and (2.41) to obtain

$$
C^{(3)}=-\frac{1}{18}\left[\begin{array}{lll}
1 & 0 & 0 \\
1 & 0 & 0 \\
1 & 0 & 0
\end{array}\right]
$$

Considering the remaining cases, we see that (2.19), (2.14), (2.15), and (2.16) imply

$$
\begin{aligned}
& B^{(5)}=-\frac{1}{6 \sqrt{6}}\left[\begin{array}{lll}
0 & 0 & 1 \\
0 & 0 & 1 \\
0 & 0 & 1
\end{array}\right], \\
& B^{(7)}=\frac{1}{6 \sqrt{6}}\left[\begin{array}{lll}
1 & 0 & 0 \\
1 & 0 & 0 \\
1 & 0 & 0
\end{array}\right], \\
& B^{(8)}=\frac{1}{6 \sqrt{6}}\left[\begin{array}{lll}
0 & 1 & 0 \\
0 & 1 & 0 \\
0 & 1 & 0
\end{array}\right], \\
& B^{(9)}=\frac{1}{6 \sqrt{6}}\left[\begin{array}{lll}
0 & 0 & 1 \\
0 & 0 & 1 \\
0 & 0 & 1
\end{array}\right],
\end{aligned}
$$

respectively, while (2.42)-(2.43), (2.32)-(2.33), (2.34)-(2.35), and (2.36)-(2.37) imply

$$
\begin{aligned}
& C^{(5)}=-\frac{1}{18}\left[\begin{array}{lll}
1 & 0 & 0 \\
1 & 0 & 0 \\
1 & 0 & 0
\end{array}\right], \\
& C^{(7)}=-\frac{1}{18}\left[\begin{array}{lll}
0 & 1 & 0 \\
0 & 1 & 0 \\
0 & 1 & 0
\end{array}\right], \\
& C^{(8)}=-\frac{1}{18}\left[\begin{array}{lll}
0 & 0 & 1 \\
0 & 0 & 1 \\
0 & 0 & 1
\end{array}\right], \\
& C^{(9)}=-\frac{1}{18}\left[\begin{array}{lll}
0 & 1 & 0 \\
0 & 1 & 0 \\
0 & 1 & 0
\end{array}\right],
\end{aligned}
$$

respectively. 
Notice that the calculation of $B^{(j)} B^{(j) *}+C^{(j)} C^{(j) *}$ for $j=3,5,7,8,9$ is identical to the calculation for $j=1$. We have

$$
B^{(j)} B^{(j) *}+C^{(j)} C^{(j) *}=\frac{5}{648}\left[\begin{array}{lll}
1 & 1 & 1 \\
1 & 1 & 1 \\
1 & 1 & 1
\end{array}\right], \quad j=3,5,7,8,9,
$$

and therefore

$$
\left\|A^{(j)}\right\|^{2}=\frac{5}{216}, \quad j=3,5,7,8,9 .
$$

Combining this with (4.11) and (4.15), we see that

$$
\sum_{j=1}^{9}\left\|A^{(j)}\right\|^{2}=\frac{13}{72}
$$

It follows from the $N=1$ case of (3.36) that

$$
\left\|T_{1}\right\| \leq \frac{1}{12} \sqrt{26}
$$

Hence

$$
\left\|T_{1} e^{i \omega}+T_{1}^{*} e^{-i \omega}\right\| \leq 2\left\|T_{1}\right\| \leq \frac{1}{6} \sqrt{26}
$$

so we have finally established (3.11). This yields

$$
\widehat{T}(\omega) \geq 1-\frac{1}{6} \sqrt{26} \approx 0.150164
$$

which is also a lower bound on the overlap matrix $S$.

We now pursue a refinement of this estimation. From our calculation of the matrices $B^{(j)}$ and $C^{(j)}$, it is an easy observation that

$$
\begin{gathered}
C^{(j)} B^{\left(j^{\prime}\right)}= \begin{cases}-\frac{1}{18} B^{\left(j^{\prime}\right)}, & j \neq 2,4,6, \\
\frac{1}{6 \sqrt{6}} B^{\left(j^{\prime}\right)}, & j=2,4,6,\end{cases} \\
C^{(j)} C^{\left(j^{\prime}\right)}= \begin{cases}-\frac{1}{18} C^{\left(j^{\prime}\right)}, & j \neq 2,4,6, \\
\frac{1}{6 \sqrt{6}} C^{\left(j^{\prime}\right)}, & j=2,4,6 .\end{cases}
\end{gathered}
$$

It follows from (4.4) that

$$
A^{(j)} A^{\left(j^{\prime}\right)}= \begin{cases}-\frac{1}{18} A^{\left(j^{\prime}\right)}, & j \neq 2,4,6 \\ \frac{1}{6 \sqrt{6}} A^{\left(j^{\prime}\right)}, & j=2,4,6\end{cases}
$$


so by (3.36) we have

$$
\begin{aligned}
\left\|T_{1}^{N}\right\|^{2} & \leq\left(\prod_{k=1}^{N} \sum_{j_{k}=1}^{9}\right) \kappa_{j_{1}}^{2} \kappa_{j_{2}}^{2} \cdots \kappa_{j_{N-1}}^{2}\left\|A^{\left(j_{N}\right)}\right\|^{2} \\
& =\left(\sum_{j=1}^{9} \kappa_{j}^{2}\right)^{N-1} \sum_{j_{N}=1}^{9}\left\|A^{\left(j_{N}\right)}\right\|^{2}, \\
\kappa_{j} & = \begin{cases}-\frac{1}{18}, & j \neq 2,4,6, \\
\frac{1}{6 \sqrt{6}}, & j=2,4,6 .\end{cases}
\end{aligned}
$$

Since

$$
\sum_{j=1}^{9} \kappa_{j}^{2}=\frac{7}{216},
$$

equation (4.22), together with (4.28), implies

$$
\left\|T_{1}^{N}\right\|^{2} \leq\left(\frac{7}{216}\right)^{N-1} \frac{13}{72}
$$

or rather

$$
\left\|T_{1}^{N}\right\| \leq\left(\frac{1}{36} \sqrt{42}\right)^{N-1} \frac{1}{12} \sqrt{26}
$$

Thus

$$
\begin{aligned}
\left\|T_{1} e^{i \omega}+T_{1}^{*} e^{-i \omega}\right\| & \leq \frac{1}{12} \sqrt{26}+\frac{1}{36} \sqrt{42} \\
& \approx 0.6049387
\end{aligned}
$$

by the standard operator lemma stated in the introduction. We have the improvement

$$
\widehat{T}(\omega) \geq 1-\frac{1}{12} \sqrt{26}-\frac{1}{36} \sqrt{42} \approx 0.3950613
$$

on the underestimate (4.25).

\section{Acknowledgment}

This work was supported in part by the National Science Foundation under Grant no. DMS-0204663. 
528 Osiris wavelets and Set wavelets

\section{References}

[1] G. Battle, Complete sets of osiris wavelets in two and three dimensions, preprint, 2002.

[2] - From a hierarchical cutoff to a hierarchical approximation, preprint, 2003.

[3] - Osiris wavelets in three dimensions, Ann. Physics 286 (2000), no. 1, 23-107.

[4] Osiris wavelets and the dipole gas, Wavelet Transforms and Time-Frequency Signal Analysis (L. Debnath, ed.), Appl. Numer. Harmon. Anal., Birkhäuser Boston, Massachusetts, 2001, pp. 81-120.

[5] - Osiris wavelets and Isis variables, Wavelets and Signal Processing (L. Debnath, ed.), Appl. Numer. Harmon. Anal., Birkhäuser Boston, Massachusetts, 2003, pp. 55-102.

[6] J. Dixmier, Les Algèbres d'Opérateurs dans l'Espace Hilbertien (Algèbres de von Neumann), Cahiers Scientifiques, vol. 25, Gauthier-Villars, Paris, 1957.

Guy Battle: Department of Mathematics, Texas A\&M University, College Station, TX 77843, USA E-mail address: battle@math.tamu.edu 


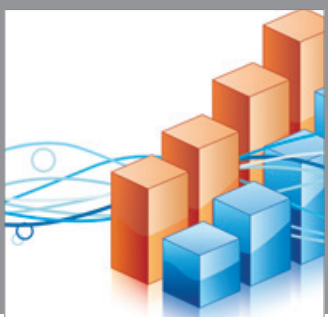

Advances in

Operations Research

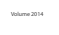

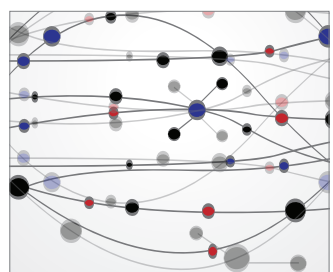

\section{The Scientific} World Journal
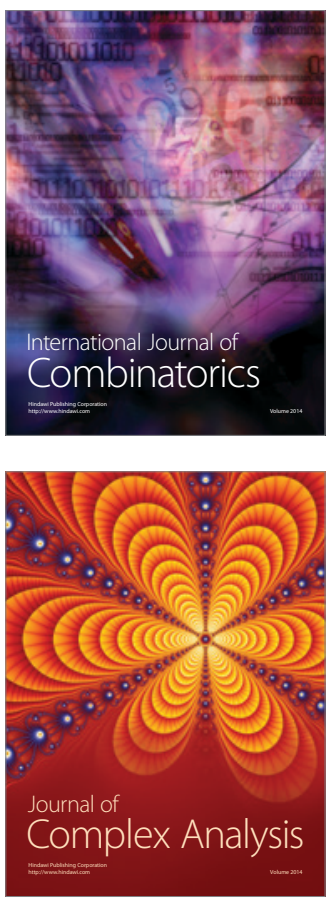

International Journal of

Mathematics and

Mathematical

Sciences
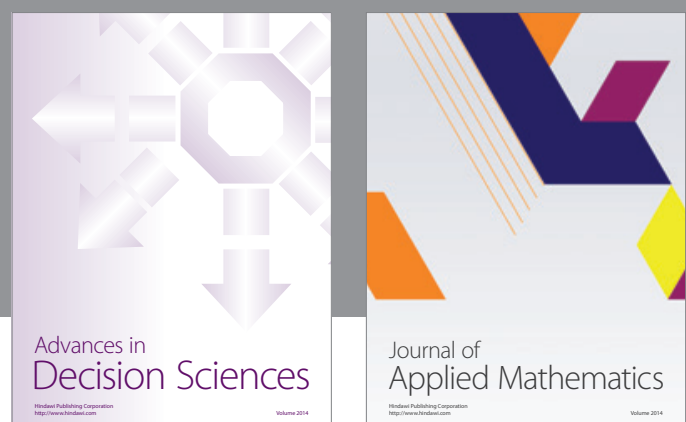

Journal of

Applied Mathematics
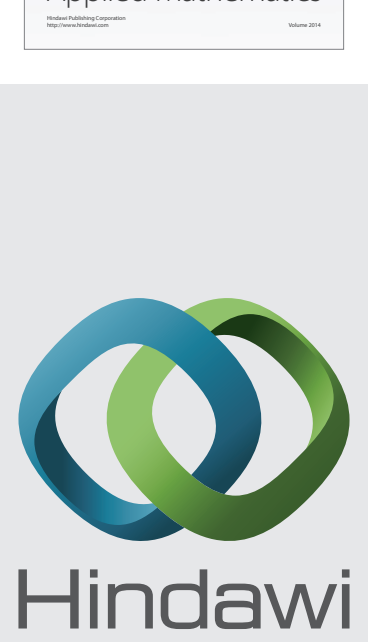

Submit your manuscripts at http://www.hindawi.com
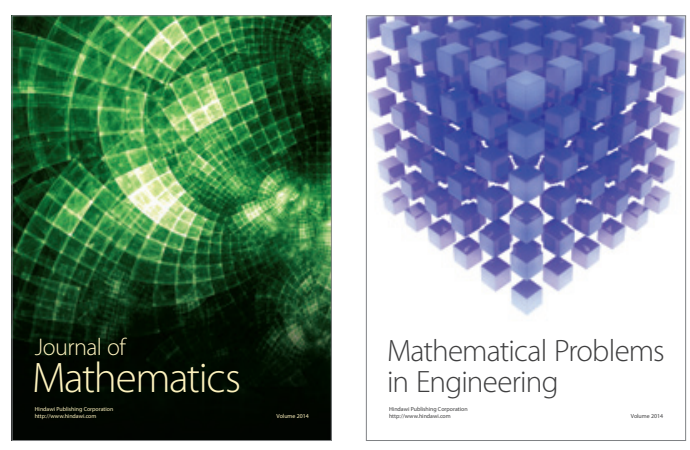

Mathematical Problems in Engineering
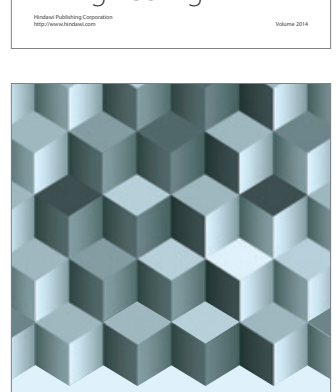

Journal of

Function Spaces
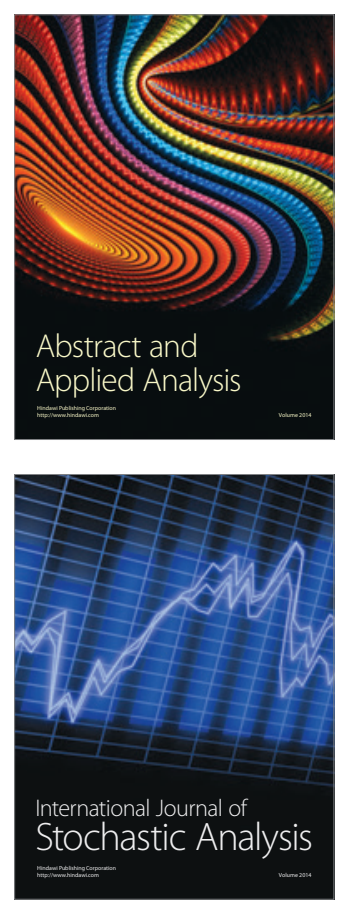

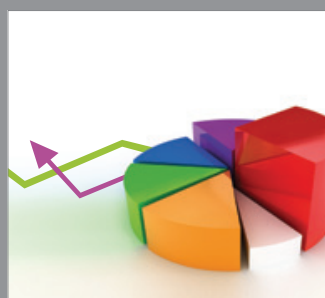

ournal of

Probability and Statistics

Promensencen
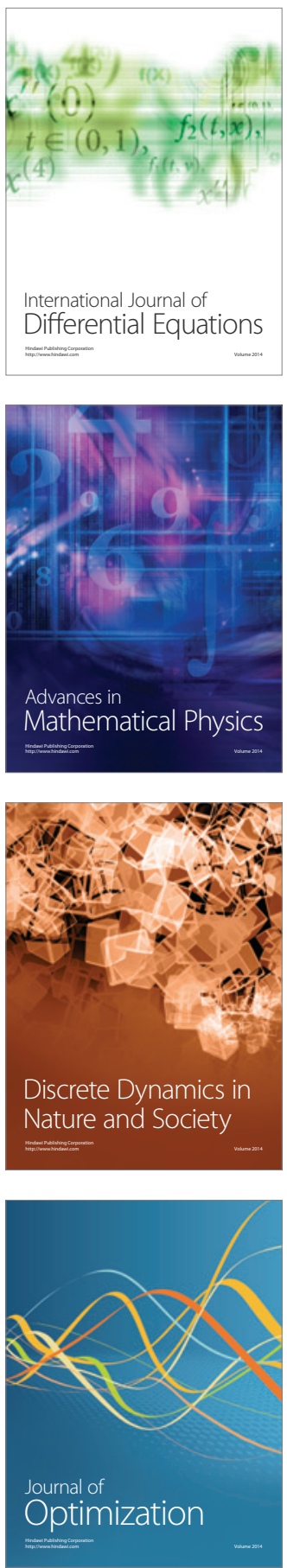